Review Article

\title{
Efficiency of Stem Cell (SC) Differentiation into Insulin- Producing Cells for Treating Diabetes: a Systematic Review
}

\author{
Marzieh Nemati $\mathbb{D}^{1}{ }^{1}$ GolamHossein Ranjbar Omrani, ${ }^{1}$ Bahareh Ebrahimi $\mathbb{D}^{2}{ }^{2}$ \\ and Aliakbar Alizadeh $(\mathbb{D})^{3,4}$ \\ ${ }^{1}$ Endocrinology and Metabolism Research Center, Shiraz University of Medical Sciences, Shiraz, Iran \\ ${ }^{2}$ Shiraz Geriatric Research Center, Shiraz University of Medical Sciences, Shiraz, Iran \\ ${ }^{3}$ Department of Tissue Engineering and Applied Cell Sciences, School of Advance Medical Science and Technology, Shiraz University \\ of Medical Sciences, Shiraz, Iran \\ ${ }^{4}$ Nanobiology and Nanomedicine Research Center, Shiraz University of Medical Sciences, Shiraz, Iran
}

Correspondence should be addressed to Aliakbar Alizadeh; alializadeha@gmail.com

Received 7 November 2020; Revised 8 February 2021; Accepted 15 February 2021; Published 26 February 2021

Academic Editor: Yohei Hayashi

Copyright (C 2021 Marzieh Nemati et al. This is an open access article distributed under the Creative Commons Attribution License, which permits unrestricted use, distribution, and reproduction in any medium, provided the original work is properly cited.

\begin{abstract}
Over the recent years, the use of stem cells has provided a new opportunity to treat various disorders including diabetes. Stem cells are unspecialized cells with a capacity for self-renewal and differentiation into more specialized cell types. Many factors contribute to the differentiation of SCs and thus play an important role in regulating the fate of stem cells. Accordingly, a wide range of protocols has been used to differentiate SCs to insulin-producing cells but the effectiveness of SC differentiation varies. The aim of this systematic review was to evaluate the results obtained from different studies on SC differentiation for higher efficacy to treat diabetes. This search was done in PubMed, Web of Science (WOS), and Scopus using keywords "insulin-producing cell (IPC)," "pancreatic B cell," "insulin-secreting cell," "stem cell," "progenitor cells," "mother cell," and "colony-forming unit." Among more than 3646 papers, 32 studies were considered eligible for more evaluations. The obtained results indicated that most of the studies were performed on the mesenchymal stem cells (MSCs) derived from different tissues as compared with other types of SCs. Different evaluations of in vitro studies as well as animal models supported their role in the recovery of diabetes. In the present review, we summarize and discuss recent advances in increasing the efficiency of SC differentiation using different materials, but despite the promising results of this systematic review, further studies are needed to assess the efficiency and safety of transplantation of these cells in diabetes recovery.
\end{abstract}

\section{Introduction}

Diabetes, as one of the most common chronic metabolic diseases, is considered a global health problem that threatens the well-being of a significant number of people across the world. Numerous data showed that the prevalence of diabetes is increasing [1], and the number of diabetics is expected to be doubled by 2025 [2]. Dietary control, regular blood sugar monitoring, oral antidiabetic drugs, and insulin injections are now the main diabetes treatments. These routines are not able to restore the precise and physiological function of beta cells and could cause long-term side effects $[3,4]$. Islet transplantation is a new treatment that can significantly reduce the insulin dependence and restore glucose homeostasis; it is also considered as an effective treatment for diabetes. However, the lack of islets and suppression of the transplanted islets by the recipient's immune system have prevented the widespread use of this treatment [5], so researchers are motivated to find other treatments. The use of stem cells (SCs) and their differentiation into beta cells is a therapeutic strategy, which has received much attention in recent years. SCs are unspecialized cells with a capacity for self-renewal and differentiation into more specialized cell types [6]. Several factors may affect the differentiation of SCs such as extracellular matrix (ECM) proteins, cell-to-cell adhesion, cell-to-cell contact, and soluble factors [7]. A wide range of protocols have been used to differentiate SCs to insulin-producing cells. However, the efficiency of differentiation and the normal 


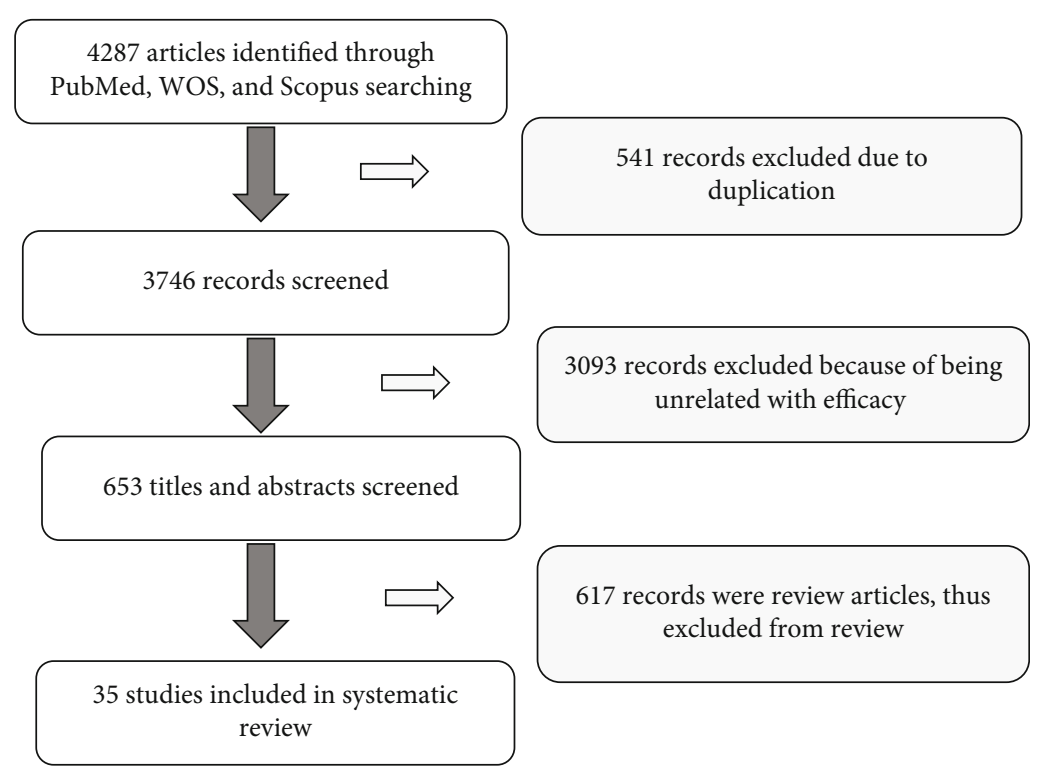

FIGURE 1: Literature search and study selection flowchart.

function of these newly formed $\beta$-cells, especially in response to glucose stimulation, are challengeable questions. The aim of this systematic review is to investigate the efficacy of differentiation of different SC sources and various interventions toward insulin-producing cells (IPCs). The results obtained from the present review could contribute to the assessment of translation of SC therapy in clinical trials.

\section{Materials and Methods}

2.1. Focused Question. This systematic review was performed to address the following question: "which intervention has the greatest effect on the differentiation efficacy of SCs into IPCs?"

2.2. Search and Study Selection. Keywords and abstract terms included ((Insulin-Producing Cell [Title/Abstract] OR Pancreatic $\beta$-cells [Title/Abstract]) OR Insulin Secreting Cell [Title/Abstract]) AND (((Stem Cell [Title/Abstract] OR Progenitor Cells [Title/Abstract]) OR Mother Cell [Title/Abstract]) OR Colony-Forming Unit [Title/Abstract]). The search strategy was applied to PubMed database, WOS, and Scopus, while focusing on the in vitro and animal model studies, and the inclusion criterion was the English language publications (by June 2020). The abstracts not published as full manuscripts, reviews, or the SC therapy studies for diseases other than diabetes were excluded. Data were collected from the full text articles as follows: (i) the source of SCs, (ii) type of the study (in vitro or in vivo), (iii) methods used for the evaluation of SC differentiation efficacy, and (iv) the obtained results.

\section{Results}

Search results and the characteristics of the included studies yielded 4287 studies. Among them, 3746 papers met all inclu- sion criteria and were selected after removing duplications. Moreover, 653 papers were reviewed and excluded (Figure 1).

By studying these article abstracts, only 35 articles included the results of the SC differentiation efficacy assay in vitro conditions (Table 1), and eight articles were related to the evaluation of treatment efficacy in animal models (Table 2). The extraction of data related to these studies is shown in Tables 1 and 2. Results revealed that the investigated SC cells have different sources. The SCs derived from embryo (ESCs) were used in twelve studies; bone marrow mesenchymal stem cells (BM-MSCs) were employed in six studies, whereas two studies investigated adipose tissuederived mesenchymal stem cells (ADMSCs), four papers examined umbilical cord-derived mesenchymal stem cells (UCMSCs), and two articles discussed Warton jelly mesenchymal stem cells (WJMSCs) for the treatment of diabetes. Induced pluripotent stem cells (iPSCs) were used in five studies; only one study examined the pancreas stem cells and one study investigated human pluripotent stem cells (hPSCs). By all these source variations, only four different methods were used and these different methods can have an important effect on the SC differentiation efficacy. In the following section, these studies are discussed according to the method used.

3.1. Use of Transfection/Transduction for Differentiation of SCs into IPCs. During embryonic development, several transcription factors are involved in the beta cell differentiation. Pancreas/duodenum homeobox protein $1\left(\mathrm{Pdx}_{1}\right)$ is one of the essential and crucial transcription factors for pancreatic development [8]. Paired box gene $4\left(\mathrm{Pax}_{4}\right)$ is another crucial regulator of pancreas development, since the lack of $\mathrm{Pax}_{4}$ activity prevents the formation of mature pancreatic insulin-producing cells [9]. Moreover, neurogenin-3( $\left.\mathrm{Ngn}_{3}\right)$, MafA, and NeuroD are the main inducers of pancreatic endocrine-specific differentiation from its primordium [10, 11]. Therefore, different studies used various transcription 


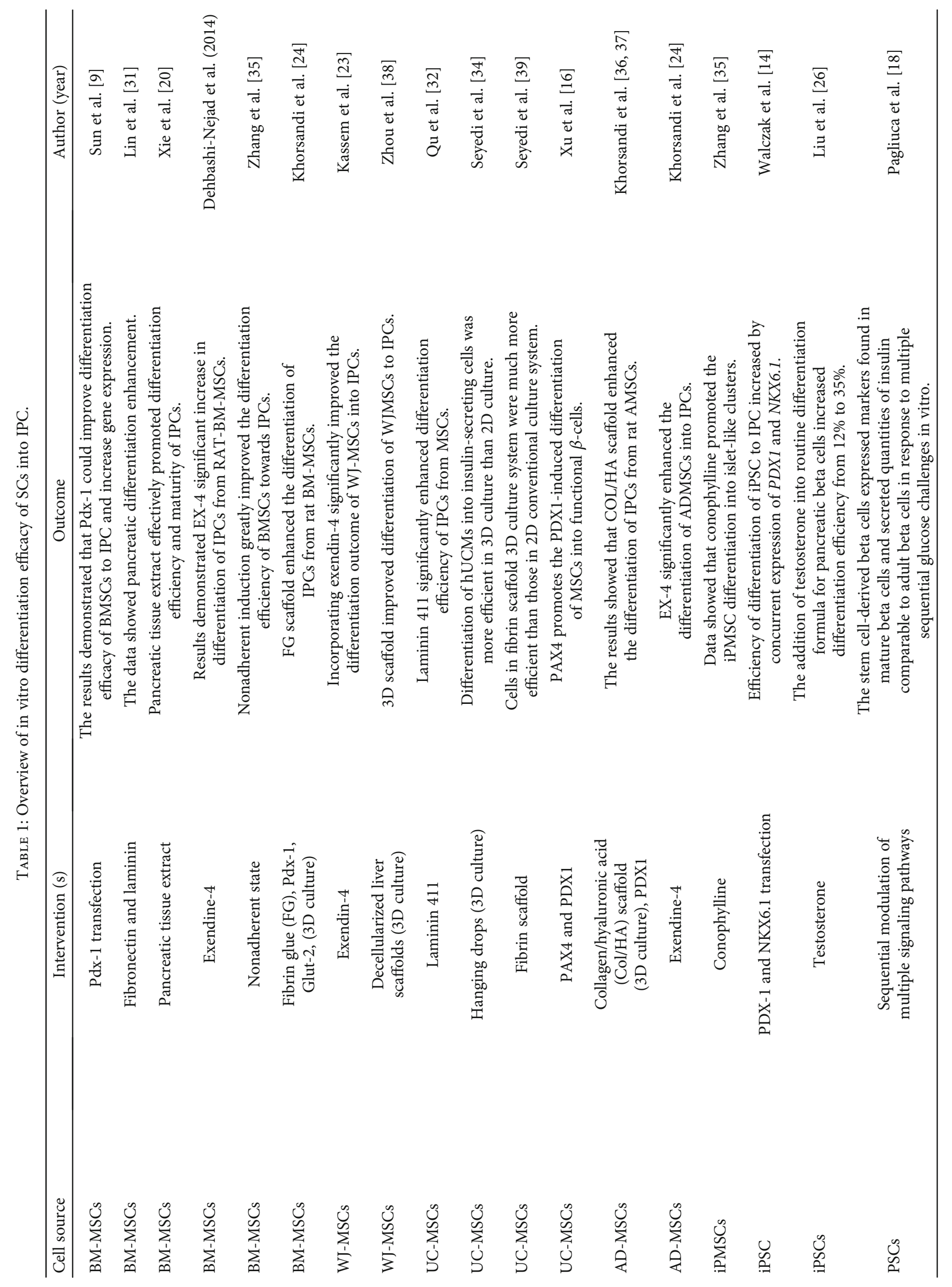




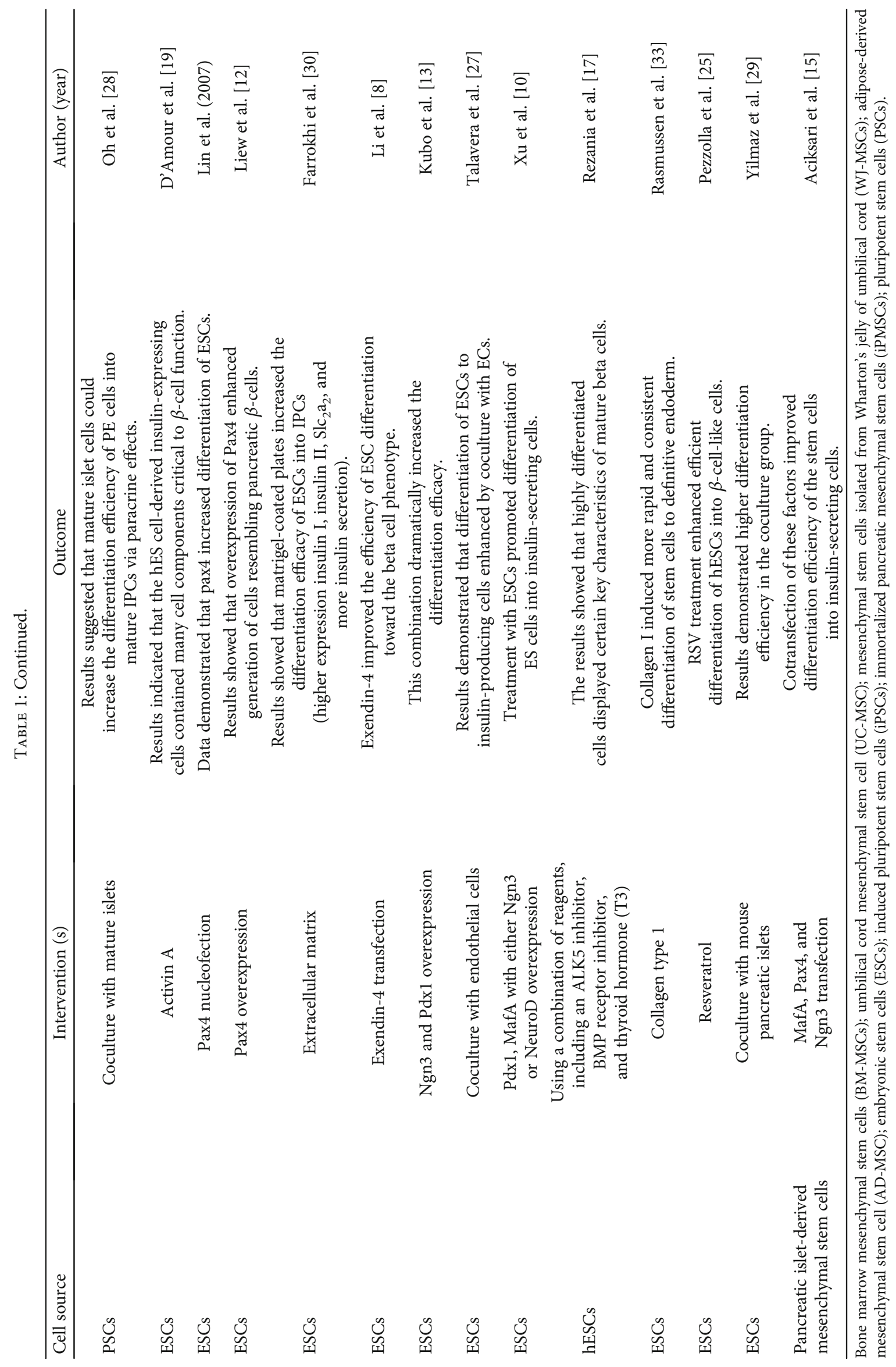




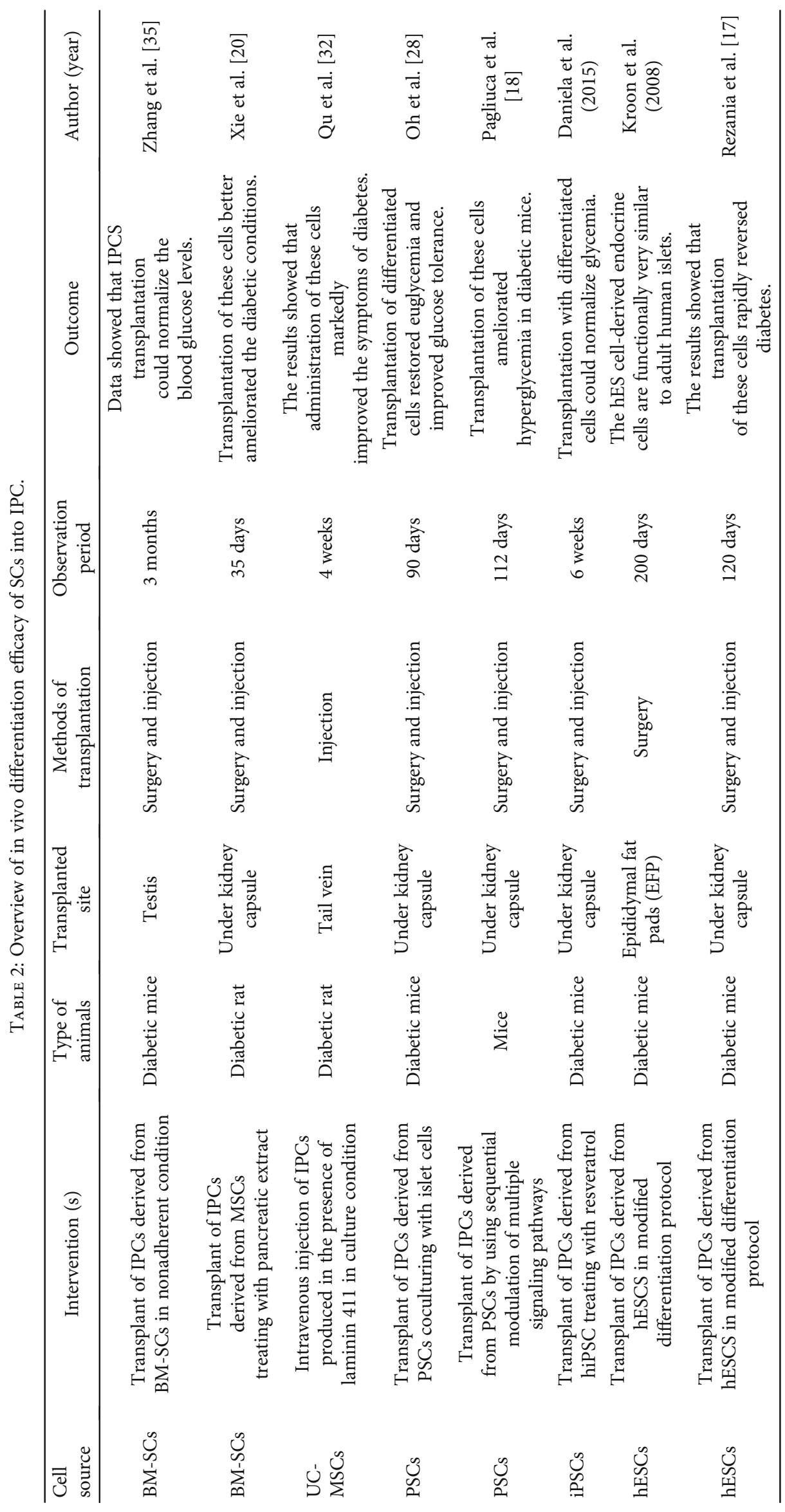


factors to increase SC differentiation efficacy into IPCs. For example, Sun et al. (2006) transduced BMSCs using the eukaryotic expression vector containing Pdx-1. The immunocytochemical expression of genes in $\mathrm{Pdx}_{1}(+)$ BMSCs were higher than that of $\mathrm{Pdx}_{1}(-) \mathrm{BMSCs}$; also, glucose-induced insulin secretion from $\mathrm{Pdx}_{1}(+) \mathrm{BMSC}$ was twofold more than that of $\mathrm{Pdx}_{1}(-) \mathrm{BMSCs}$ [9].

In two other studies, the researchers demonstrated that the overexpression of $\mathrm{Pax}_{4}$ using transfection could produce more mature IPCs from ESCs $[9,12] . \mathrm{Pax}_{4}$ and $\mathrm{Ngn}_{3}$ transfection into murine ESCs also resulted in a significant upregulation of insulin genes (Ins1 and Ins2) and other pancreasrelated genes and consequently generated efficient IPCs [13]. Also, expression of $\mathrm{Pdx}_{1}$ and MafA with either $\mathrm{Ngn}_{3}$ or NeuroD could significantly increase the differentiation efficiency of ESCs into IPCs. These observations were confirmed by qRT-PCR, immunocytochemistry, and ELISA assay [10]. Studies had shown that $\mathrm{pdx}_{1}$ and $\mathrm{NKX}_{6.1}$ gene transfection could increase the differentiation efficiency of induced pluripotent SCs to IPC and the obtained IPCs secreted significantly higher insulin and C-peptide levels [14]. Overexpression of $\operatorname{Pax}_{4}$ and MafA also resulted in ameliorating the differentiation efficiency of the pancreatic islet mesenchymal stem cells (PiMSCs) into IPCs [15]. The last study which used this method showed that the upregulation of $\mathrm{Pax}_{4}$ and $\mathrm{Pdx}_{1}$ could markedly enhance the differentiation of MSCs to form mature islet-like clusters and functional insulin-producing $\beta$-like cells [16].

\subsection{Treatment Approaches Used for Differentiation of SCs} into IPCs. Different combinations were used in eleven studies to increase the differentiation of SCs into IPCs. Hua et al. (2010) treated mouse ESCs by high concentration of exendin-4. The expression pattern of IPC markers showed that exendin- 4 could improve the efficiency of differentiation of SCs toward the $\beta$-cell phenotype [8]. Pagliuca and Rezania found that using the modified differentiation protocol improved the differentiation efficiency of ESCs [17] and PSCs [18] into IPC. In addition, adding activin A to the cultural condition of hESCs could effectively produce beta cells [19]. Another study showed that BM-MSC treatment with pancreatic extract improved the differentiation efficiency and maturity of IPCs [20]. Zhang et al. (2013) demonstrated that using conophylline promoted induced pluripotent mesenchymal stem cell (iPMSC) differentiation into IPCs [21]. BMSCs [22], WJMSCs [23], and ADMSC [24] treatment with exendin-4 improved the differentiation outcome of these SCs to IPCs. Pezzolla et al. found that treating human embryonic stem cells (hESCs) with resveratrol improved the differentiation efficiency, produced numerous insulin-positive cells, and induced significantly higher $\mathrm{Pdx}_{1}$ expression [25]. Interestingly, addition of testosterone into the differentiation formula for pancreatic $\beta$-cells could increase the differentiation efficiency of human-induced pluripotent stem cells (hiPSCs) into IPCs from $12 \%$ to $35 \%$. The administration of testosterone promoted the expression of key genes associated with $\beta$-cell differentiation including $\mathrm{Ngn}_{3}, \mathrm{NeuroD}_{1}$, and INS [26].
3.3. Coculture Method Used for Differentiation of SCs into IPCs. Various studies have used different substances in SC coculturing in order to increase the differentiation efficacy of SCs into IPCs. For instance, using endothelial cell coculture for enhancing ESC differentiation has been reported only in one study, revealing that endothelial cells enhanced embryonic stem cell differentiation to pancreatic progenitors and IPCs through bone morphogenetic protein (BMP) signaling [27]. Bea et al. (2015) cultured pluripotent SCs in the presence of mature islet cells. They found that the paracrine effects of these cells could lead to an increase in the differentiation efficiency of the pancreatic endoderm (PE) cells into mature IPCs [28]. A study performed by Yilmaz et al. (2015) revealed that the ESC coculture with mouse pancreatic islets resulted in higher differentiation efficacy toward IPCs [29].

3.4. Use of Extracellular Matrix Proteins for Differentiation of SCs into IPCs. In 2007, Farrokhi et al. evaluated the effect of extracellular matrices (ECMs) on their differentiation to IPCs. RT-PCR and also secretory function analysis of their finding showed higher expression of $\beta$-cell-specific markers including insulin I, insulin II, Slc $\mathrm{a}_{2}$, and more insulin secretion in the matrigel-coated plate group [30]. In two other studies, using fibronectin, laminin [31], and laminin 411 [32] promoted the differentiation of MSCs into insulinproducing cells and had upregulated insulin expression at both mRNA and protein levels. Rasmussen et al. (2015) also reported that treating SCs by ECM proteins in a particular collagen type I with fibronectin could improve the differentiation of human ESCs toward definitive endoderm [33].

3.5. Use of In Vivo Microenvironmental Simulation for Differentiation of SCs into IPCs. Seyedi et al. (2015) used a $3 \mathrm{D}$ culture condition (hanging drop) to differentiate hUCMs into IPC. RT-PCR and immunohistochemistry analysis showed higher expression of insulin protein and insulin production in hanging drops than the monolayer group [34]. RT-PCR, immunofluorescence, and radioimmunoassay (RIA) in another study revealed the improvement in the differentiation efficiency of BMSCs toward IPCs under a nonadherent state [35]. Evaluation using a 3D culture to differentiate ADMSCs [36, 37], BMSCs [36, 37], WJMSCs $[33,38]$, and human umbilical mesenchymal stem cells (hUCMSCs) [39] by different evaluation methods, such as RT-PCR, immunofluorescence, RIA, and ELISA assay, showed more than a threefold increase in the differentiation efficacy compared to the $2 \mathrm{D}$ experimental culture cell usage.

3.6. Animal Studies Performed for the Treatment of Diabetes by Transplantation of IPC Differentiated from SCs. The efficacy of SC-derived IPC therapy on diabetes was evaluated in eight different animal studies. Xie et al. transplanted IPCs derived from MSCs treated with pancreatic extract under the right kidney capsule of streptozotocin- (STZ-) induced diabetic rats to evaluate the ability of these cells in the treatment of diabetes. They observed that IPCs were able to release more insulin in a glucose-dependent manner and ameliorate the diabetic conditions of STZ-treated rats better [20]. In 


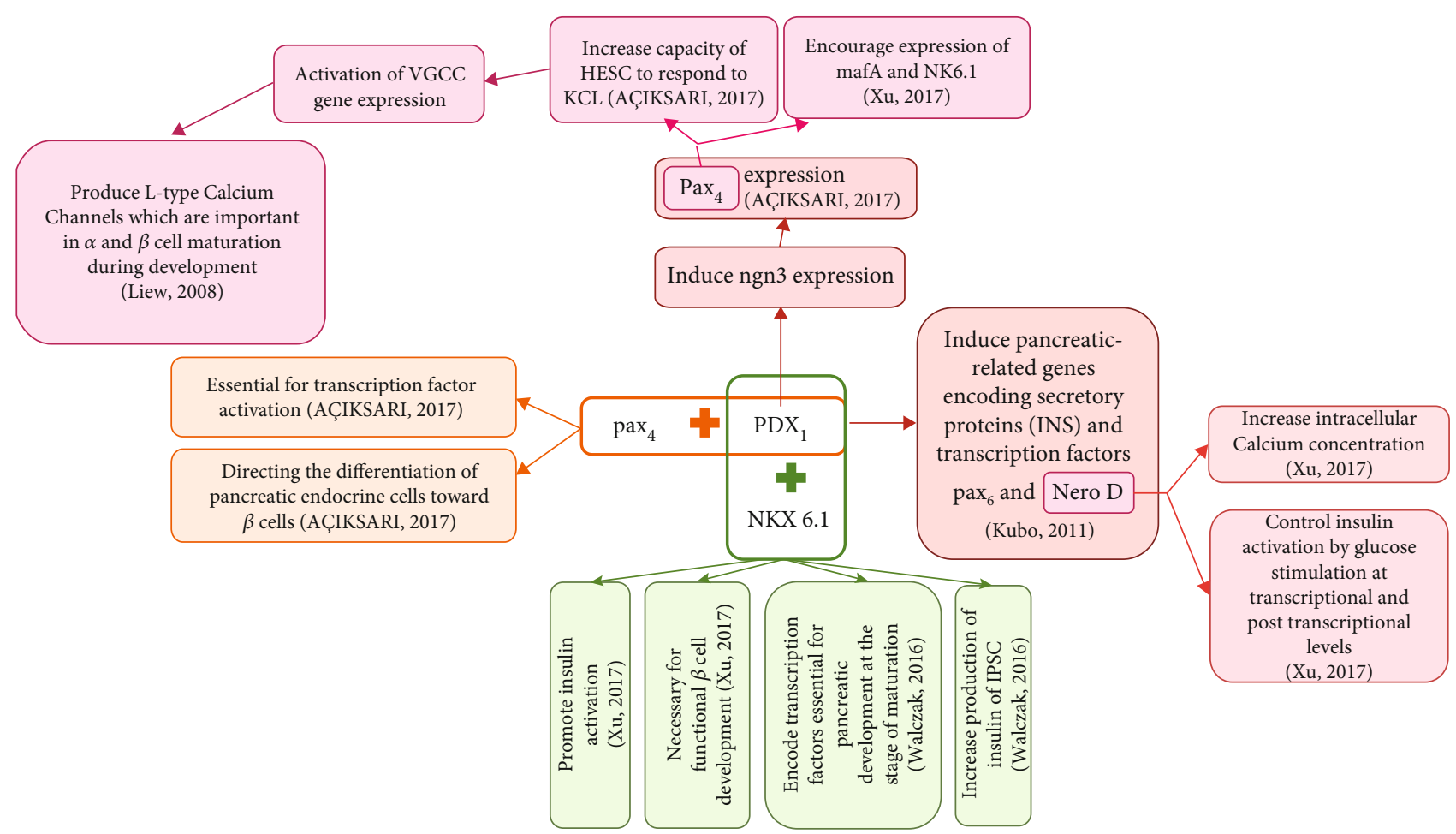

FIGURE 2: Summary of different pathways increasing beta cell differentiation.

other studies, the hESC-derived islet-like clusters were used and injected under the kidney capsule or the epididymal fat pad could transiently normalize glycaemia in diabetic mice ([25], Kroon et al. 2008, [17]). Other studies investigated the effect of differentiation efficacy of pancreatic islet-like cell clusters derived from hBMSCs under nonadherent induction by transplanting differentiated cells to diabetic mice. They found that these cells normalized hyperglycemia in diabetic mice [35]. Another study on diabetic nude mice indicated that transplantation diabetic mice with IPCs derived from pancreatic stem cells cocultured with islet cells exhibited restored euglycemia and improved glucose tolerance [28]. Qu et al. (2014) also revealed that administration of laminin 411-induced IPCs rapidly and significantly downregulated fasting blood glucose levels, significantly reduced the $\mathrm{HbA}_{1} \mathrm{c}$ concentration, and markedly improved the symptoms and survival of diabetic rats [32]. Pagliuca and his colleagues found that transplantation of IPCs derived from PSCs by using sequential modulation of multiple signaling pathways ameliorated hyperglycemia in diabetic mice [18].

\section{Discussion}

This study is the first systematic review about the efficacy of SC differentiation toward IPCs in restoration of diabetes. This review included the results of in vitro differentiation and animal studies. The various sources of SCs used in these studies and their different evaluation methods highlight the advantage of comparing the obtained results with each other. In most studies, BM-MSCs and ESCs were used under variable conditions to differentiate into IPCs for assessing the best differentiation efficacy in diabetes therapy. The results of these studies indicated that the transfected stem cells with IPC key transcription factors, including $\mathrm{PDX}_{1}, \mathrm{PAX}_{4}, \mathrm{MafA}$, $\mathrm{NKX}_{6.1}$, and NeuroD, could improve the differentiation efficacy of SCs into IPCs. As the results of various studies have shown, the use of these essential factors is considered as an effective factor in SC differentiation into IPCs $([8,9,12$, 13], Xie, Wang et al. 2013, [14, 15]). Furthermore, it appears that the treatment of SCs with different factors plays a key role in the improvement of differentiation efficacy and subsequently on the therapeutic outcome.

The comparison of the obtained results indicated that the type of treatments could influence the efficacy of differentiation through diverse pathways (Figure 2). Some factors may improve it via stimulating the expression of islet-associated genes ( $\mathrm{Pdx}_{1}$ and insulin), leading to increased insulin release upon glucose challenge, as demonstrated by Hua et al. (2010) [8], Nejad-Dehbashi et al. (2013) [22], and others [14, 36, 37]. Rat pancreatic extract has been used as a differentiation inducer because it contains pancreatic development and differentiation proteins that could improve the differentiation efficacy of BM-MSCs and also ameliorate the diabetic conditions of streptozotocin- (STZ-) treated rats [20]. In addition, the result of another study showed that conophylline stimulated iPMSC proliferation and promoted their potential differentiation into an islet-like cluster [21]. In three studies, the coculture technique was used to increase the differentiation efficacy of SCs into IPCs [27-29]. The results obtained from these three studies indicated that the differentiation of SCs into insulin-producing cells under coculture conditions with endothelial cells or islet cells were effectively increased. Additionally, an in vivo study showed that the diabetic mice transplanted with differentiated SC coculturing with islet 
cells exhibited restored euglycemia and improved glucose tolerance [28].

Several research groups tried to examine the effect of different extracellular matrix proteins on stem cell differentiation efficacy. The results obtained by Hsiao-Yun et al. indicated that IPC differentiation by MSCs could be enhanced by adding ECM and these stimulatory effects were mediated through activation of Akt and ERK pathways [31]. The use of laminin 411 by Huiting et al. (2014), as a differentiation inducer of IPCs from UC-MSCs via the $\mathrm{Pdx}_{1}$ and $\mathrm{Ngn}_{3}$ signaling pathways, efficiently improved the differentiation, symptoms, and survival of diabetic rats [32]. Another study performed by Rasmussen et al. (2015) demonstrated that collagen I could effectively differentiate the embryonic SCs into functional $\beta$-cells [33]. The evaluation of stem cell differentiation efficacy on 3D culture environments has been performed in five studies [34-37, 39]. A 3D culture is advantageous to imitate the in vivo microenvironment by enhancing cell-cell and cell-matrix interactions and the subsequent cell signaling $[40,41]$. In line with this fact, the results obtained by Zhang (2014), Khorsandi et al. (2014), Khorsandi et al. and (2015) showed that nonadherent induction [35], collagen/hyaluronic acid (Col/HA) scaffold [36, 37], and FG scaffolds $[36,37]$ could greatly promote SCs to form pancreatic islet-like cell clusters and improve the differentiation efficiency of SCs toward IPCs.

\section{Conclusion}

In summary, the results obtained from this systematic review provide better understanding about the efficacy of SC differentiation into IPCs for treating diabetes. The obtained results showed that the IPCs derived from SCs by imitating the in vivo microenvironment and using $3 \mathrm{D}$ culture showed a better efficacy than the other methods of differentiation to restore diabetes. However, the obtained results indicate that more evaluation is needed about the efficacy of SC differentiation into IPCs for the treatment of diabetes.

\section{Conflicts of Interest}

The authors declare that they have no competing interest.

\section{References}

[1] A. Stokes and S. H. Preston, "Deaths attributable to diabetes in the United States: comparison of data sources and estimation approaches," PLoS One, vol. 12, no. 1, p. e0170219, 2017.

[2] F. S. Samani, M. Ebrahimi, T. Zandieh et al., "In vitro differentiation of human umbilical cord blood CD133+ cells into insulin producing cells in co-culture with rat pancreatic mesenchymal stem cells," Cell Journal (Yakhteh), vol. 17, no. 2, p. 211, 2015.

[3] M. D. Bellin, F. B. Barton, A. Heitman et al., "Potent induction immunotherapy promotes long-term insulin independence after islet transplantation in type 1 diabetes," American Journal of Transplantation, vol. 12, no. 6, pp. 1576-1583, 2012.

[4] A. J. Shapiro, J. R. T. Lakey, E. A. Ryan et al., "Islet transplantation in seven patients with type 1 diabetes mellitus using a glucocorticoid-free immunosuppressive regimen," New
England Journal of Medicine, vol. 343, no. 4, pp. 230-238, 2000.

[5] S. Porat and Y. Dor, "New sources of pancreatic beta cells," Current Diabetes Reports, vol. 7, no. 4, pp. 304-308, 2007.

[6] M. Wei, S. Li, and W. Le, "Nanomaterials modulate stem cell differentiation: biological interaction and underlying mechanisms," Journal of Nanobiotechnology, vol. 15, no. 1, p. 75, 2017.

[7] B. Xu, D. Fan, Y. Zhao et al., "Three-dimensional culture promotes the differentiation of human dental pulp mesenchymal stem cells into insulin-producing cells for improving the diabetes therapy," Frontiers in Pharmacology, vol. 10, 2020.

[8] H. Li, A. Lam, A. M. Xu, K. Sl Lam, and S. Kim Chung, "High dosage of exendin- 4 increased early insulin secretion in differentiated beta cells from mouse embryonic stem cells," Acta Pharmacologica Sinica, vol. 31, no. 5, pp. 570-577, 2010.

[9] J. Sun, Y. Yang, X. Wang, J. Song, and Y. Jia, “Expression of $\mathrm{Pdx}-1$ in bone marrow mesenchymal stem cells promotes differentiation of islet-like cells in vitro," Science in China Series C: Life Sciences, vol. 49, no. 5, pp. 480-489, 2006.

[10] H. Xu, K. S. Tsang, J. C. N. Chan et al., "The combined expression ofPdxlandMafAwith EitherNgn3orNeuroDimproves the differentiation efficiency of mouse embryonic stem cells into insulin-producing cells," Cell Transplantation, vol. 22, no. 1, pp. 147-158, 2013.

[11] X. Xu, J. D'Hoker, G. Stange et al., " $\beta$ cells can be generated from endogenous progenitors in injured adult mouse pancreas," Cell, vol. 132, no. 2, pp. 197-207, 2008.

[12] C. G. Liew, N. N. Shah, S. J. Briston et al., "PAX4 enhances beta-cell differentiation of human embryonic stem cells," PLoS One, vol. 3, no. 3, p. e1783, 2008.

[13] A. Kubo, R. Stull, M. Takeuchi et al., "Pdx1 and Ngn3 overexpression enhances pancreatic differentiation of mouse ES cellderived endoderm population," PLoS One, vol. 6, no. 9, p. e24058, 2011.

[14] M. P. Walczak, A. M. Drozd, E. Stoczynska-Fidelus, P. Rieske, and D. P. Grzela, "Directed differentiation of human iPSC into insulin producing cells is improved by induced expression of PDX1 and NKX6. 1 factors in IPC progenitors," Journal of Translational Medicine, vol. 14, no. 1, p. 341, 2016.

[15] A. AÇIKSARI, G. DURUKSU, and E. KARAÖZ, "Improved insulin-secreting properties of pancreatic islet mesenchymalstem cells by constitutive expression of Pax4 and MafA," Turkish Journal of Biology, vol. 41, no. 6, pp. 979-991, 2017.

[16] L. Xu, C. Xu, S. Zhou et al., "PAX4 promotes PDX1-induced differentiation of mesenchymal stem cells into insulinsecreting cells," American Journal of Translational Research, vol. 9, no. 3, pp. 874-886, 2017.

[17] A. Rezania, J. E. Bruin, P. Arora et al., "Reversal of diabetes with insulin-producing cells derived_in vitro_from human pluripotent stem cells," Nature Biotechnology, vol. 32, no. 11, pp. 1121-1133, 2014.

[18] F. W. Pagliuca, J. R. Millman, M. Gürtler et al., "Generation of functional human pancreatic $\beta$ cells in vitro," Cell, vol. 159, no. 2, pp. 428-439, 2014.

[19] K. A. D'Amour, A. G. Bang, S. Eliazer et al., "Production of pancreatic hormone-expressing endocrine cells from human embryonic stem cells," Nature Biotechnology, vol. 24, no. 11, pp. 1392-1401, 2006.

[20] H. Xie, Y. Wang, H. Zhang, H. Qi, H. Zhou, and F.-R. Li, "Role of injured pancreatic extract promotes bone marrow-derived 
mesenchymal stem cells efficiently differentiate into insulinproducing cells," PLoS One, vol. 8, no. 9, p. e76056, 2013.

[21] H.-r. ZHANG, L. I. Dan, H. CAO et al., "Conophylline promotes the proliferation of immortalized mesenchymal stem cells derived from fetal porcine pancreas (iPMSCs)," Journal of Integrative Agriculture, vol. 12, no. 4, pp. 678-686, 2013.

[22] F. Nejad-Dehbashi, M. Hashemitabar, M. Orazizadeh, S. Bahramzadeh, E. S. Pourshoushtary, and L. Khorsandi, "The effects of exendine- 4 on insulin producing cell differentiation from rat bone marrow-derived mesenchymal stem cells," Cell Journal (Yakhteh), vol. 16, no. 2, p. 187, 2014.

[23] D. H. Kassem, M. M. Kamal, A. E.-L. G. El-Kholy, and H. O. El-Mesallamy, "Exendin-4 enhances the differentiation of Wharton's jelly mesenchymal stem cells into insulinproducing cells through activation of various $\beta$-cell markers," Stem Cell Research \& Therapy, vol. 7, no. 1, p. 108, 2016.

[24] L. Khorsandi, S. Saremy, A. Khodadadi, and F. Dehbashi, "Effects of exendine- 4 on the differentiation of insulin producing cells from rat adipose-derived mesenchymal stem cells," Cell Journal (Yakhteh), vol. 17, no. 4, p. 720, 2016.

[25] D. Pezzolla, J. Lopez-Beas, C. C. Lachaud et al., "Resveratrol ameliorates the maturation process of $\beta$-cell-like cells obtained from an optimized differentiation protocol of human embryonic stem cells," PLoS One, vol. 10, no. 3, p. e0119904, 2015.

[26] H. Liu, D. Guo, A. Ruzi et al., "Testosterone improves the differentiation efficiency of insulin-producing cells from human induced pluripotent stem cells," PLoS One, vol. 12, no. 6, p. e0179353, 2017.

[27] D. Talavera-Adame, G. Wu, Y. He et al., "Endothelial cells in co-culture enhance embryonic stem cell differentiation to pancreatic progenitors and insulin-producing cells through BMP signaling," Stem Cell Reviews and Reports, vol. 7, no. 3, pp. 532-543, 2011.

[28] B. J. Oh, S.-H. Oh, J. M. Choi et al., "Co-culture with mature islet cells augments the differentiation of insulin-producing cells from pluripotent stem cells," Stem Cell Reviews and Reports, vol. 11, no. 1, pp. 62-74, 2015.

[29] I. Yilmaz, A. Sariboyaci, C. Subasi, and E. Karaoz, "Differentiation potential of mouse embryonic stem cells into insulin producing cells in pancreatic islet microenvironment," Experimental and Clinical Endocrinology \& Diabetes, vol. 124, no. 2, pp. 120-129, 2016.

[30] A. Farrokhi, E. Elahi, M. Pirouz, H. Jafary, and H. Baharvand, "Effect of extracellular matrix on differentiation of mouse embryonic stem cells into insulin-producing cells," Yakhteh Medical Journal, vol. 10, no. 4, pp. 250-259, 2009.

[31] H.-Y. Lin, C.-C. Tsai, L.-L. Chen, S.-H. Chiou, Y.-J. Wang, and S.-C. Hung, "Fibronectin and laminin promote differentiation of human mesenchymal stem cells into insulin producing cells through activating Akt and ERK," Journal of Biomedical Science, vol. 17, no. 1, p. 56, 2010.

[32] H. Qu, X. Liu, Y. Ni et al., "Laminin 411 acts as a potent inducer of umbilical cord mesenchymal stem cell differentiation into insulin-producing cells," Journal of Translational Medicine, vol. 12, no. 1, p. 135, 2014.

[33] C. H. Rasmussen, D. R. Petersen, J. B. Moeller, M. Hansson, and M. Dufva, "Collagen type I improves the differentiation of human embryonic stem cells towards definitive endoderm," PLoS One, vol. 10, no. 12, p. e0145389, 2015.

[34] F. Seyedi, A. Farsinejad, S. A. Nematollahi-Mahani, T. Eslaminejad, and S. N. Nematollahi-Mahani, "Suspension culture alters insulin secretion in induced human umbilical cord matrix-derived mesenchymal cells," Cell Journal (Yakhteh), vol. 18, no. 1, p. 52, 2016.

[35] Y. Zhang and Z. Dou, "Under a nonadherent state, bone marrow mesenchymal stem cells can be efficiently induced into functional islet-like cell clusters to normalize hyperglycemia in mice: a control study," Stem Cell Research \& Therapy, vol. 5, no. 3, p. 66, 2014.

[36] L. Khorsandi, A. Khodadadi, F. Nejad-Dehbashi, and S. Saremy, "Three-dimensional differentiation of adiposederived mesenchymal stem cells into insulin-producing cells," Cell and Tissue Research, vol. 361, no. 3, pp. 745-753, 2015.

[37] L. Khorsandi, F. Nejad-Dehbashi, A. Ahangarpour, and M. Hashemitabar, "Three-dimensional differentiation of bone marrow-derived mesenchymal stem cells into insulinproducing cells," Tissue and Cell, vol. 47, no. 1, pp. 66-72, 2015.

[38] P. Zhou, Y. Guo, Y. Huang et al., "The dynamic threedimensional culture of islet-like clusters in decellularized liver scaffolds," Cell and Tissue Research, vol. 365, no. 1, pp. 157$171,2016$.

[39] F. Seyedi, A. Farsinejad, and S. N. Nematollahi-Mahani, "Fibrin scaffold enhances function of insulin producing cells differentiated from human umbilical cord matrix-derived stem cells," Tissue and Cell, vol. 49, no. 2, pp. 227-232, 2017.

[40] W. L. Grayson, T. Ma, and B. Bunnell, "Human mesenchymal stem cells tissue development in 3D PET matrices," Biotechnology Progress, vol. 20, no. 3, pp. 905-912, 2004.

[41] H. Wang, T. Brun, K. Kataoka, A. J. Sharma, and C. B. Wollheim, "MAFA controls genes implicated in insulin biosynthesis and secretion," Diabetologia, vol. 50, no. 2, pp. 348-358, 2007. 